\title{
Maximum GCD Among Pairs of Random Integers
}

\author{
R. W. R. Darling \& E. E. Pyle \\ Mathematics Research Group, National Security Agency \\ 9800 Savage Road, Fort George G. Meade, Maryland 20755-6515
}

November 2, 2018

\begin{abstract}
ABSTRACT: Fix $\alpha>0$, and sample $N$ integers uniformly at random from $\left\{1,2, \ldots,\left\lfloor e^{\alpha N}\right\rfloor\right\}$. Given $\eta>0$, the probability that the maximum of the pairwise GCDs lies between $N^{2-\eta}$ and $N^{2+\eta}$ converges to 1 as $N \rightarrow \infty$. More precise estimates are obtained. This is a Birthday Problem: two of the random integers are likely to share some prime factor of order $N^{2} / \log [N]$. The proof generalizes to any arithmetical semigroup where a suitable form of the Prime Number Theorem is valid.
\end{abstract}

\section{Main Result}

Whereas the distribution of the sizes of the prime divisors of a random integer is a well studied subject — see portions of Billingsley (1999) — the authors are unaware of any published results on the pairwise Greatest Common Divisors (GCD) among a large collection of random integers. Theorem 1.1 establishes probabilistic upper and lower bounds for the maximum of these pairwise GCDs.

\subsection{Theorem}

Suppose $\alpha>0$, and $T_{1}, \ldots, T_{N}$ is a random sample, drawn with replacement, from the integers $\left\{n \in \mathbb{N}: n \leq e^{\alpha N}\right\}$. Let $\Gamma_{j, k}$ denote the Greatest Common Divisor of $T_{j}$ and $T_{k}$. For any $\eta>0$,

$$
\lim _{N \rightarrow \infty} \mathbb{P}\left[N^{2-\eta}<\max _{1 \leq j<k \leq N}\left\{\Gamma_{j, k}\right\}<N^{2+\eta}\right]=1 .
$$

Indeed there are more precise estimates: for all $s \in(0,1)$, and $b>0$, the right side of (2) is finite, and

$$
\mathbb{P}\left[\max _{1 \leq j<k \leq N}\left\{\Gamma_{j, k}\right\} \geq N^{2 / s} b^{1 / s}\right] \leq \frac{1}{2 b} \prod_{p \in \mathcal{P}}\left(1+\frac{p^{s}-1}{p^{2}-p^{s}}\right),
$$

where $\mathcal{P}$ denotes the rational primes; while if $\Lambda_{j, k}$ denotes the largest common prime factor of $T_{j}$ and $T_{k}$, then for all $\theta>0$, 


$$
\lim _{N \rightarrow \infty} \mathbb{P}\left[\max _{1 \leq k<j \leq N}\left\{\Lambda_{j, k}\right\}<\frac{N^{2}}{\log \left[N^{\theta}\right]}\right] \leq e^{-\theta / 8} .
$$

Supplement: There is an upper bound, similar to (2), for the radical (i.e the largest square-free divisor) $\operatorname{rad}\left[\Gamma_{j, k}\right]$ of the GCD:

$$
\mathbb{P}\left[\max _{1 \leq j<k \leq N}\left\{\operatorname{rad}\left[\Gamma_{j, k}\right]\right\} \geq N^{2 / s} b^{1 / s}\right] \leq \frac{1}{2 b} \prod_{p \in \mathcal{P}}\left(1-p^{-2}+p^{s-2}\right) .
$$

The proof, which is omitted, uses methods similar to those of Proposition 2.2, based upon a Bernoulli model for occurrence of prime divisors, instead of a Geometric model for prime divisor multiplicities. For example, when $s=0.999$, the product on the right side of (4) is approximately 12.44; for the right side of (2), it is approximately 17.64 .

\subsection{Overview of the Proof of Theorem 1.1}

Let $Z_{i}^{k}$ be a Bernoulli random variable, which takes the value 1 when prime $p_{i}$ divides $T_{k}$. As a first step towards the proof, imagine proving a comparable result in the case where $\left\{Z_{i}^{k}, 1 \leq k \leq N, i \geq 1\right\}$ were independent, and $\mathbb{P}\left[Z_{i}^{k}=1\right]=1 / p_{i}$. The harder parts of the proof arise in dealing with the reality that, for fixed $k,\left\{Z_{i}^{k}, i \geq 1\right\}$ are negatively associated, and change with $N$. Convergence of the series

$$
\sum_{p \in \mathcal{P}} p^{-2} \log [p]<\infty
$$

ensures that the parameter $\alpha$, which governs the range of integers being sampled, appears neither in (1), (2), nor (3). However the proof for the lower bound depends crucially on an exponential (in $N$ ) rate of growth in the range, in order to moderate the dependence among $\left\{Z_{i}^{k}, i \geq 1\right\}$ for fixed $k$.

Consider primes as labels on a set of urns; the random variable $T_{j}$ contributes a ball to the urn labelled $p$ if prime $p$ divides $T_{j}$. The lower bound comes from showing that, with asymptotic probability at least $1-e^{-\theta / 8}$, some urn with a label $p>N^{2} / \log \left[N^{\theta}\right]$ contains more than one ball; in that case prime $p$ is a common divisor of two distinct members of the list $T_{1}, \ldots, T_{N}$. The upper bound comes from an exponential moment inequality.

If $T_{1}, \ldots, T_{N}$ were sampled uniformly without replacement from the integers from 1 to $N^{2}$, the lower bound (3) would fail; see the analysis in Billingsley (1999) of the distribution of the largest prime divisor of a random integer. In the case of sampling from integers from 1 to $N^{r}$, where $r \geq 3$, the upper bound (2) remains valid, but we do not know whether the lower bound (3) holds or not. 


\subsection{Generalizations to Arithmetical Semigroups}

Although details will not be given, the techniques used to prove Theorem 1.1 will be valid in the more general context of a commutative semigroup $G$ with identity element 1 , containing a countably infinite subset $\mathcal{P}:=\left\{p_{1}, p_{2}, \ldots\right\}$ called the primes of $G$, such that every element $a \neq 1$ of $G$ has a unique factorization of the form

$$
a=\prod_{i \geq 1} p_{i}^{e_{i}},\left(e_{1}, e_{2}, \ldots\right) \in \mathbb{Z}_{+}^{\infty}
$$

where all but finitely many $\left(e_{i}\right)$ are zero. Assume in addition that $G$ is an arithmetical semigroup in the sense of Knopfmacher (1990), meaning that there exists a real-valued norm $|\cdot|$ on $G$ such that:

- $|1|=1,\left|p_{i}\right|>1$ for all $p_{i} \in \mathcal{P}$.

- $|a b|=|a||b|$ for all $a, b \in G$.

- The set $\pi_{G}[x]:=\left\{i \geq 1:\left|p_{i}\right| \leq e^{x}\right\}$ is finite, for each real $x>0$.

The only analytic condition needed is an abstract form of the Prime Number Theorem (see Knopfmacher (1990), Chapter 6):

$$
\lim _{x \rightarrow \infty} x e^{-x}\left|\pi_{G}[x]\right|=1,
$$

used in the proof of Proposition 4.1. This in turn will imply convergence of series such as:

$$
\sum_{p \in \mathcal{P}} \log \left[1+|p|^{s-2}\right], s<1,
$$

which appear (in an exponentiated form) in the bound (2). For example, Landau's Prime Ideal Theorem provides such a result in the case where $G$ is the set of integral ideals in an algebraic number field, $\mathcal{P}$ is the set of prime ideals, and $|a|$ is the norm of $a$. Knopfmacher (1990) also studies a more general setting where, for some $\delta>0$,

$$
\lim _{x \rightarrow \infty} x e^{-\delta x}\left|\pi_{G}[x]\right|=\delta .
$$

The authors have not attempted to modify Theorem 1.1 to fit this case.

\section{Pairwise Minima in a Geometric Probability Model}

\subsection{Geometric Random Vectors}

Let $\mathcal{P}:=\left\{p_{1}, p_{2}, \ldots\right\}$ denote the rational primes $\{2,3,5, \ldots\}$ in increasing order. Let $\mathcal{I}$ denote the set of non-negative integer vectors $\left(e_{1}, e_{2}, \ldots\right)$ for which $\sum e_{i}<\infty$. Let $X_{1}, X_{2}, \ldots$ be (possibly dependent) positive integer random 
variables, whose joint law has the property that, for every $k \in \mathbb{N}$. and every $\left(e_{1}, e_{2}, \ldots\right) \in \mathcal{I}$ for which $e_{k}=0$,

$$
\mathbb{P}\left[X_{k} \geq m \mid \underset{i \neq k}{\cap}\left\{X_{i}=e_{i}\right\}\right] \leq\left(\frac{1}{p_{k}}\right)^{m} .
$$

Let $\zeta$ denote the random vector:

$$
\zeta:=\left(X_{1}, X_{2}, \ldots\right) \in \mathbb{N}^{\mathbb{N}} .
$$

Consider the finite-dimensional projections of $X_{1}, X_{2}, \ldots$ as a general model for prime multiplicities in the prime factorization of a random integer, without specifying exactly how that integer will be sampled. Let $\zeta^{(1)}, \zeta^{(2)}, \ldots, \zeta^{(N)}$ be independent random vectors, all having the same law as $\zeta$ in (6). Write $\zeta^{(k)}$ as $\left(X_{1}^{k}, X_{2}^{k}, \ldots\right)$. Then

$$
L_{j, k}:=\sum_{i} \min \left\{X_{i}^{k}, X_{i}^{j}\right\} \log \left[p_{i}\right]
$$

is a model for the log of the GCD of two such random integers. We shall now derive an upper bound for

$$
\Delta_{N}:=\max _{1 \leq k<j \leq N}\left\{L_{j, k}\right\},
$$

which models the log maximum of the pairwise GCD among a set of $N$ "large, random" integers.

\subsection{Proposition}

Assume the joint law of the components of $\zeta$ satisfies (5).

(i) For every $s \in(0,1)$, the following expectation is finite:

$$
\mathbb{E}\left[e^{s L_{k, j}}\right]<\prod_{i}\left(1+\frac{p_{i}^{s}-1}{p_{i}^{2}-p_{i}^{s}}\right)=: C_{s}<\infty, s<1 .
$$
bound:

(ii) For any $s \in(0,1)$, and $b>C_{s} / 2$, for $C_{s}$ as in (7), there is an upper

$$
\mathbb{P}\left[\Delta_{N} \geq \log \left[N^{2 / s}\right]+s^{-1} \log [b]\right] \leq \frac{C_{s}}{2 b}<1 .
$$

Proof: Consider first the case where $X_{1}, X_{2}, \ldots$ are independent Geometric random variables, and

$$
\mathbb{P}\left[X_{k} \geq m\right]=\left(\frac{1}{p_{k}}\right)^{m}, m=1,2, \ldots
$$

It is elementary to check that, for $s \in(0,1)$, and any $p \in \mathcal{P}$, if $X^{\prime \prime}, X^{\prime}$ are independent Geometric random variables with 


$$
\mathbb{P}\left[X^{\prime \prime} \geq m\right]=p^{-m}=\mathbb{P}\left[X^{\prime} \geq m\right], m=1,2, \ldots,
$$

then their minimum is also a Geometric random variable, which satisfies

$$
\mathbb{E}\left[p^{s \min \left\{X^{\prime \prime}, X^{\prime}\right\}}\right]=1+\frac{p^{s}-1}{p^{2}-p^{s}}<1+p^{s-2} .
$$

It follows from the independence assumption that

$$
\mathbb{E}\left[e^{s L_{k, j}}\right]=\mathbb{E}\left[\prod_{i} p_{i}^{s \min \left\{X_{i}^{k}, X_{i}^{j}\right\}}\right]=\prod_{i}\left(1+\frac{p_{i}^{s}-1}{p_{i}^{2}-p_{i}^{s}}\right)=C_{s} .
$$

This verifies the assertion (7). Markov's inequality shows that, for any $s \in(0,1)$

$$
C_{s} \geq e^{s t} \mathbb{P}\left[L_{k, j} \geq t\right] .
$$

Furthermore

$$
\begin{gathered}
\mathbb{P}\left[\max _{1 \leq k<j \leq N}\left\{L_{k, j}\right\} \geq t\right]=\mathbb{P}\left[{\left.\underset{1 \leq k<j \leq N}{\cup}\left\{L_{k, j} \geq t\right\}\right]}^{\leq} \sum_{1 \leq k<j \leq N} \mathbb{P}\left[L_{k, j} \geq t\right]=\frac{N(N-1)}{2} \mathbb{P}\left[L_{k, j} \geq t\right] .\right.
\end{gathered}
$$

It follows that, for $s \in(0,1), b>0$, and $t:=s^{-1} \log \left[b N^{2}\right]$

$$
\mathbb{P}\left[\Delta_{N} \geq \log \left[N^{2 / s}\right]+s^{-1} \log [b]\right] \leq \frac{N^{2}}{2} e^{-s t} C_{s}=\frac{C_{s}}{2 b} .
$$

It remains to consider the case where $X_{1}, X_{2}, \ldots$ satisfies (5), without the independence assumption. Choose a probability space $(\Omega, \mathcal{F}, \mathbb{P})$ on which independent Geometric random variables $X_{1}^{\prime}, X_{2}^{\prime}, \ldots$ and $X_{1}^{\prime \prime}, X_{2}^{\prime \prime}, \ldots$ are defined, such that for all $i \geq 1$,

$$
\mathbb{P}\left[X_{i}^{\prime \prime} \geq m\right]=p_{i}^{-m}=\mathbb{P}\left[X_{i}^{\prime} \geq m\right], m=1,2, \ldots
$$

We propose to construct $\zeta^{(1)}=\left(X_{1}^{1}, X_{2}^{1}, \ldots\right)$ and $\zeta^{(2)}=\left(X_{1}^{2}, X_{2}^{2}, \ldots\right)$ by induction, on this probability space $(\Omega, \mathcal{F}, \mathbb{P})$, so that for each $n \geq 1$, $\left\{\left(X_{i}^{1}, X_{i}^{2}\right) 1 \leq i \leq n\right\}$ have the correct joint law, and

$$
X_{i}^{1} \leq X_{i}^{\prime} ; X_{i}^{2} \leq X_{i}^{\prime \prime}, i=1,2, \ldots .
$$

Once this is achieved, monotonicity implies

$$
\mathbb{E}\left[e^{s L_{1,2}}\right] \leq \mathbb{E}\left[\prod_{i} p_{i}^{s \min \left\{X_{i}^{\prime}, X_{i}^{\prime \prime}\right\}}\right],
$$

so the desired result will follow from the previous one for independent Geometric random variables. 
Since $\zeta^{(1)}$ and $\zeta^{(2)}$ are independent, it suffices to construct $\zeta^{(1)}$ in terms of $X_{1}^{\prime}, X_{2}^{\prime}, \ldots$ so that $X_{i}^{1} \leq X_{i}^{\prime}$ for all $i$. Let $\left(U_{i, j}, i \geq 1, j \geq 0\right)$ be independent Uniform $(0,1)$ random variables. Suppose either $i=1$, or else some values $X_{1}^{1}=$ $e_{1}, X_{2}^{1}=e_{2}, \ldots, X_{i-1}^{1}=e_{i-1}$ have already been determined. By assumption, there exists parameters

$$
q_{i, k}:=\mathbb{P}\left[X_{i} \geq k \mid \bigcap_{j<i}\left\{X_{j}=e_{j}\right\}\right] \leq\left(\frac{1}{p_{i}}\right)^{k}, k=1,2, \ldots
$$

Use these to construct $X_{i}^{\prime}$ and $X_{i}^{1}$ as follows:

$$
\begin{aligned}
X_{i}^{\prime} & :=\min \left\{k: U_{i, 0} U_{i, 1} \ldots U_{i, k}>\left(\frac{1}{p_{i}}\right)^{k}\right\} ; \\
X_{i}^{1} & :=\min \left\{k: U_{i, 0} U_{i, 1} \ldots U_{i, k}>q_{i, k}\right\} \leq X_{i}^{\prime} .
\end{aligned}
$$

This completes the construction and the proof, giving the result (8).

\section{Lower Bound for Largest Collision}

\subsection{Random Vectors with Independent Components}

Let $\mathcal{P}:=\left\{p_{1}, p_{2}, \ldots\right\}$ denote the rational primes $\{2,3,5, \ldots\}$ in increasing order, and let $a_{j}:=\left(\log \left[p_{j}\right]\right)^{1 / 2}$. Instead of the Geometric model (5), switch to a Bernoulli model in which $Z_{1}, Z_{2}, \ldots$ are independent Bernoulli random variables, with

$$
\mathbb{P}\left[Z_{j}=1\right]:=\frac{1}{p_{j}} .
$$

Let $\xi$ denote the random vector

$$
\xi:=\left(a_{1} Z_{1}, a_{2} Z_{2}, \ldots\right) \in[0, \infty)^{\mathbb{N}} .
$$

under this new assumption, and let $\xi^{(1)}, \xi^{(2)}, \ldots, \xi^{(N)}$ be independent random vectors, all having the same law as $\xi$. Note that $\xi^{(1)} \cdot \xi^{(2)}$ is not a suitable model for the GCD of two random integers, because the independence assumption (9) is not realistic. However it is a useful context to develop the techniques which will establish the lower bound in Theorem 1.1.

Write $\xi^{(k)}=\left(a_{1} Z_{1}^{k}, a_{2} Z_{2}^{k}, \ldots\right)$. We seek a lower bound on the log of the largest prime $p_{i}$ at which a "collision" occurs, meaning that $Z_{i}^{j}=1=Z_{i}^{k}$ for some $j, k$ :

$$
\Delta_{N}^{\prime}:=\max _{1 \leq k<j \leq N}\left\{\max _{i}\left\{Z_{i}^{j} Z_{i}^{k} \log \left[p_{i}\right]\right\} \leq \max _{1 \leq k<j \leq N}\left\{\xi^{(k)} \cdot \xi^{(j)}\right\}\right.
$$




\subsection{Proposition}

Given $\delta \in(0, \infty)$, define $\varphi_{N}:=\varphi_{N}[\delta]$ implicitly by the identity

$$
\int_{\varphi_{N}}^{2 \varphi_{N}} \frac{N^{2} d x}{2 x^{2} \log [x]}=\delta
$$

Under the assumption of independence of the components of the random vector (10),

$$
\lim _{N \rightarrow \infty} \mathbb{P}\left[\Delta_{N}^{\prime} \geq \log \left[\varphi_{N}[\delta]\right]\right] \geq 1-e^{-\delta} .
$$

Remark: From the integration bounds:

$$
\frac{1}{2 \varphi_{N} \log \left[2 \varphi_{N}\right]}=\frac{1}{\log \left[2 \varphi_{N}\right]} \int_{\varphi_{N}}^{2 \varphi_{N}} \frac{d x}{x^{2}}<\frac{2 \delta}{N^{2}}<\frac{1}{\log \left[\varphi_{N}\right]} \int_{\varphi_{N}}^{2 \varphi_{N}} \frac{d x}{x^{2}}=\frac{1}{2 \varphi_{N} \log \left[\varphi_{N}\right]}
$$

it follows that $\varphi_{N}$, defined in (11), satisfies $\varphi_{N} \log \left[\varphi_{N}\right] / N^{2} \rightarrow 0.25 / \delta$. Hence for all sufficiently large $N, \varphi_{N}<N^{2} / 2$, and

$$
\varphi_{N}>\frac{N^{2}}{4 \delta \log \left[2 \varphi_{N}\right]}>\frac{N^{2}}{8 \delta \log [N]} .
$$

The proof uses the following technical Lemma, which the reader may treat as a warm-up exercise for the more difficult Proposition 4.1.

\subsection{Lemma}

Let $\mathcal{P}_{N}$ denote the set of primes $p$ such that $\varphi_{N}<p \leq 2 \varphi_{N}$. Let $\left\{Z_{p}^{k}, p \in \mathcal{P}_{N}, 1 \leq k \leq N\right\}$ be independent Bernoulli random variables, where $\mathbb{P}\left[Z_{p}^{k}=1\right]=1 / p$. Take $D_{p}:=Z_{p}^{1}+\ldots+Z_{p}^{N} . \quad$ Then

$$
\lim _{N \rightarrow \infty} \mathbb{P}\left[\bigcup_{p \in \mathcal{P}_{N}}\left\{D_{p} \geq 2\right\}\right]=1-e^{-\delta} .
$$

Proof: Binomial probabilities give:

$$
\begin{aligned}
& \mathbb{P}\left[D_{p} \leq 1\right]=\left(1-\frac{1}{p}\right)^{N}+\frac{N}{p}\left(1-\frac{1}{p}\right)^{N-1}=\left(1-\frac{1}{p}\right)^{N}\left(1+\frac{N}{p-1}\right) \\
= & \left(1-\frac{N}{p}+\frac{N(N-1)}{2 p^{2}}-\ldots\right)\left(1+\frac{N}{p-1}\right)=1-\frac{N^{2}}{2 p^{2}}+O\left(\frac{N}{\varphi_{N}^{2}}\right)+O\left(\left(\frac{N}{\varphi_{N}}\right)^{3}\right) .
\end{aligned}
$$

Independence of $\left\{Z_{p}^{k}, p \in \mathcal{P}_{N}, 1 \leq k \leq N\right\}$ implies independence of $\left\{D_{p}, p \in \mathcal{P}_{N}\right\}$, so 


$$
\begin{gathered}
\log \left[\mathbb{P}\left[\underset{p \in \mathcal{P}_{N}}{\cap}\left\{D_{p} \leq 1\right\}\right]\right]=\sum_{p \in \mathcal{P}_{N}} \log \left[\mathbb{P}\left[D_{p} \leq 1\right]\right] \\
=\sum_{p \in \mathcal{P}_{N}} \log \left[1-\frac{N^{2}}{2 p^{2}}\right]+O\left(\frac{N\left|\mathcal{P}_{N}\right|}{\varphi_{N}^{2}}\right)+O\left(\frac{N^{3}\left|\mathcal{P}_{N}\right|}{\varphi_{N}^{3}}\right) .
\end{gathered}
$$

Using the estimates $\varphi_{N} \log \left[\varphi_{N}\right]=O\left(N^{2}\right),\left|\mathcal{P}_{N}\right|=O\left(\varphi_{N} / \log \left[\varphi_{N}\right]\right)$, and $p / \varphi_{N} \leq 2$, the last expression becomes

$$
=-\sum_{p \in \mathcal{P}_{N}} \frac{N^{2}}{2 p^{2}}+O\left(\frac{N^{2}}{\varphi_{N}^{2}}\right)+O\left(\frac{N}{\varphi_{N} \log \left[\varphi_{N}\right]}\right)+O\left(\frac{N^{3}}{\varphi_{N}^{2} \log \left[\varphi_{N}\right]}\right) .
$$

All terms but the first vanish in the limit, while the Prime Number Theorem ensures that

$$
\lim _{N \rightarrow \infty} \sum_{p \in \mathcal{P}_{N}} \frac{N^{2}}{2 p^{2}}=\delta .
$$

Therefore

$$
\lim _{N \rightarrow \infty} \mathbb{P}\left[\underset{p \in \mathcal{P}_{N}}{\cap}\left\{D_{p} \leq 1\right\}\right]=e^{-\delta}
$$

Thus the limit (14) follows.

\subsubsection{Proof of Proposition}

According to our model, if $D_{p} \geq 2$ for some $p=p_{i} \in \mathcal{P}_{N}$, then there are indices $1 \leq k<j \leq N$ for which $Z_{i}^{j}=1=Z_{i}^{k}$. Since $\log \left[p_{i}\right] \geq \log \left[\varphi_{N}[\delta]\right]$,

$$
\lim _{N \rightarrow \infty} \mathbb{P}\left[\Delta_{N}^{\prime} \geq \log \left[\varphi_{N}[\delta]\right]\right] \geq \lim _{N \rightarrow \infty} \mathbb{P}\left[\cup_{p \in \mathcal{P}_{N}}\left\{D_{p} \geq 2\right\}\right]=1-e^{-\delta}
$$

This verifies (12).

\section{Application: Pairwise GCDs of Many Uniform Random Integers}

We shall now prove an analogue of Lemma 3.3 which applies to random integers, dropping the independence assumption for the components of the random vector (10). 


\subsection{Proposition}

Suppose $\alpha>0$, and $T_{1}, \ldots, T_{N}$ is a random sample, drawn with replacement, from the integers $\left\{n \in \mathbb{N}: n \leq e^{\alpha N}\right\}$. Given $\delta \in(0, \infty)$, define $\varphi_{N}:=\varphi_{N}[\delta]$ implicitly by the identity (11) . Let $\mathcal{P}_{N}$ denote the set of primes $p$ such that $\varphi_{N}<p \leq 2 \varphi_{N} ;$ for $p \in \mathcal{P}_{N}$ let $D_{p}$ denote the number of elements of $\left\{T_{1}, \ldots, T_{N}\right\}$ which are divisible by $p$. Then

$$
\lim _{N \rightarrow \infty} \mathbb{P}\left[\bigcup_{p \in \mathcal{P}_{N}}\left\{D_{p} \geq 2\right\}\right]=1-e^{-\delta} .
$$

Proof: As noted above, the Prime Number Theorem ensures that

$$
\lim _{N \rightarrow \infty} \sum_{p \in \mathcal{P}_{N}} \frac{N^{2}}{2 p^{2}}=\delta
$$

More generally, the alternating series for the exponential function ensures that there is an even integer $d \geq 1$ such that, given $\epsilon \in(0,1)$, for all sufficiently large $N$,

$$
1-e^{-\delta /(1+\epsilon)}<\sum_{r=1}^{d}(-1)^{r+1} I_{r}<1-e^{-\delta /(1-\epsilon)}
$$

where, for $\left\{p_{1}, \ldots, p_{r}\right\} \subset \mathcal{P}_{N}$

$$
I_{r} F 39 E:=\sum_{p_{1}<\ldots<p_{r}} \frac{N^{2 r}}{2^{r}\left(p_{1} \ldots p_{r}\right)^{2}}, r=1,2, \ldots, d .
$$

Because $\varphi_{N} / N^{2} \rightarrow 0$, it follows that, for every $\left\{p_{1}, \ldots, p_{d}\right\} \subset \mathcal{P}_{N}$,

$$
\frac{p_{1} \ldots p_{d}}{e^{\alpha N}}<\frac{\left(\varphi_{N}\right)^{d}}{e^{\alpha N}}<e^{2 d \log [N]-\alpha N} \rightarrow 0 .
$$

Suppose that, for this constant value of $d$, we fix some $\left\{p_{1}, \ldots, p_{d}\right\} \subset \mathcal{P}_{N}$; instead of sampling $T_{1}, \ldots, T_{N}$ uniformly from integers up to $e^{\alpha N}$, sample $T_{1}^{\prime}, \ldots, T_{N}^{\prime}$ uniformly from integers up to

$$
p_{1} \ldots p_{d}\left\lfloor e^{\alpha N} /\left(p_{1} \ldots p_{d}\right)\right\rfloor .
$$

¿From symmetry considerations, the Bernoulli random variables $B_{1}^{\prime}, \ldots, B_{d}^{\prime}$ are independent, with parameters $1 / p_{1}, \ldots, 1 / p_{d}$, respectively where $B_{i}^{\prime}$ is the indicator of the event that $p_{i}$ divides $T_{1}^{\prime}$. By elementary reasoning,

$$
\begin{gathered}
\mathbb{P}\left[D_{p} \geq 2\right]=\frac{N^{2}}{2 p^{2}}+O\left(\left(N / \varphi_{N}\right)^{3}\right) ; \\
\mathbb{P}\left[D_{p_{1}} \geq 2, \ldots, D_{p_{r}} \geq 2\right]=\frac{N^{2 r}}{2^{r}\left(p_{1} \ldots p_{r}\right)^{2}}+O\left(\left(N / \varphi_{N}\right)^{2 r+1}\right), r=1,2, \ldots, d .
\end{gathered}
$$


If we were to sample $T_{1}, \ldots, T_{N}$ instead of $T_{1}^{\prime}, \ldots, T_{N}^{\prime}$, the most that such a probability could change is

$$
\mathbb{P}\left[\bigcup_{i=1}^{N}\left\{T_{i} \neq T_{i}^{\prime}\right\}\right] \leq \frac{N p_{1} \ldots p_{d}}{e^{\alpha N}}<e^{(2 d+1) \log [N]-\alpha N} .
$$

The same estimate holds for any choice of $\left\{p_{1}, \ldots, p_{d}\right\} \subset \mathcal{P}_{N}$. By the inclusion-exclusion formula, taken to the first $d$ terms,

$$
\begin{gathered}
\mathbb{P}\left[\bigcup_{p \in \mathcal{P}_{N}}\left\{D_{p} \geq 2\right\}\right] \geq \sum_{p \in \mathcal{P}_{N}} \mathbb{P}\left[D_{p} \geq 2\right]-\sum_{p_{1}<p_{2}} \mathbb{P}\left[D_{p_{1}} \geq 2, D_{p_{2}} \geq 2\right]+\ldots-\sum_{p_{1}<\ldots<p_{d}} \mathbb{P}\left[D_{p_{1}} \geq 2, \ldots, D_{p_{d}} \geq 2\right] \\
=\sum_{r=1}^{d}(-1)^{r+1} I_{r}+O\left(\left(N / \varphi_{N}\right)^{3}\right)+\left(\begin{array}{c}
N \\
d
\end{array}\right) e^{(2 d+1) \log [N]-\alpha N} .
\end{gathered}
$$

So under this simplified model, the reasoning above combines to show that, for all sufficiently large $N$,

$$
1-e^{-\delta /(1+\epsilon)}<\mathbb{P}\left[\bigcup_{p \in \mathcal{P}_{N}}\left\{D_{p} \geq 2\right\}\right]<1-e^{-\delta /(1-\epsilon)} .
$$

Since $\epsilon$ can be made arbitrarily small, this verifies the result.

\subsection{Proof of Theorem 1.1}

Suppose $\alpha>0$, and $T_{1}, \ldots, T_{N}$ is a random sample, drawn with replacement, from the integers $\left\{n \in \mathbb{N}: n \leq e^{\alpha N}\right\}$. Let $\Lambda_{j, k}$ denote the largest common prime factor of $T_{j}$ and $T_{k}$. Take

$$
\Delta_{N}^{\prime}:=\max _{1 \leq k<j \leq N}\left\{\log \left[\Lambda_{j, k}\right]\right\} .
$$

In the language of Proposition 4.1, if $D_{p} \geq 2$ for some $p \in \mathcal{P}_{N}$, then there are indices $1 \leq k<j \leq N$ for which $\Lambda_{j, k}>\varphi_{N}$. So inequality (13) and Proposition 4.1 imply that, for any $\theta=8 \delta>0$

$$
\begin{gathered}
\lim _{N \rightarrow \infty} \mathbb{P}\left[\Delta_{N}^{\prime} \geq 2 \log [N]-\log \left[\log \left[N^{\theta}\right]\right]\right] \geq \lim _{N \rightarrow \infty} \mathbb{P}\left[\Delta_{N}^{\prime} \geq \log \left[\varphi_{N}[\theta / 8]\right]\right] \\
\geq \lim _{N \rightarrow \infty} \mathbb{P}\left[\bigcup_{p \in \mathcal{P}_{N}}\left\{D_{p} \geq 2\right\}\right]=1-e^{-\theta / 8} .
\end{gathered}
$$

This is precisely the lower bound (3). For any $\eta>0$, the lower bound in (1) follows from:

$$
\lim _{N \rightarrow \infty} \mathbb{P}\left[\Delta_{N}^{\prime}>(2-\eta) \log [N]\right]=1
$$


Let $\Gamma_{j, k} \geq \Lambda_{j, k}$ denote the Greatest Common Divisor of $T_{j}$ and $T_{k}$. To obtain the upper bound (2) on $\Gamma_{j, k}$, it suffices by Proposition 2.2 to check that condition (5) is valid, when $X_{i}$ denotes the multiplicity to which prime $p_{i}$ divides $T_{1}$. Take any positive integer $r \geq 1$, any prime $p_{k}$ coprime to $r$, and any $m \geq 1$. The conditional probability that $p_{k}^{m}$ divides $T_{1}$, given that $r$ divides $T_{1}$, is

$$
\frac{\left\lfloor e^{\alpha N} /\left(r p_{k}^{m}\right)\right\rfloor}{\left\lfloor e^{\alpha N} / r\right\rfloor} \leq\left(\frac{1}{p_{k}}\right)^{m} .
$$

So condition (5) holds. Thus (8) holds, which is equivalent to (2).

Finally we derive the upper bound in (1), for an arbitrary $\eta>0$. Fix $\epsilon \in(0,1)$ and $\eta>0$. Select $s \in(0,1)$ to satisfy $2 / s=2+\eta / 2$. Then choose $b=C_{s}^{\prime} / \epsilon$. According to (8),

$$
\mathbb{P}\left[\Delta_{N} \geq(2+\eta / 2) \log [N]+s^{-1} \log [b]\right] \leq \epsilon / 2 .
$$

For any $N$ sufficiently large so that $(\eta / 2) \log [N]>s^{-1} \log [b]$,

$$
\mathbb{P}\left[\Delta_{N} \geq(2+\eta) \log [N]\right] \leq \epsilon / 2 .
$$

This yields the desired bound (1).

\section{References}

[1] Patrick Billingsley, Convergence of Probability Measures, Wiley, 1999.

[2] John Knopfmacher, Abstract Analytic Number Theory, Dover, New York, 1990. 